\title{
Direct detections of Majorana dark matter in vector portal
}

\author{
Wei Chao \\ Center for Advanced Quantum Studies, \\ Department of Physics, Beijing Normal University, \\ Beijing, 100875, China \\ E-mail: chaowei@bnu.edu.cn
}

ABSTRACT: In this paper we investigate the direct detections of Majorana dark matter $(\mathrm{MDM})$ in vector portal. Considering that the tree-level scattering cross sections in these models are either dark matter velocity suppressed or spin-dependent, we calculate radiative corrections to the spin-independent cross section in effective field theory approach. Wilson coefficients of effective MDM-quark interactions are calculated at the one-loop level, and the Wilson coefficient of the effective MDM-gluon interaction is derived at the two-loop level. Numerical results show that current constraints can rule out a narrow mass range of MDM when tree-level contributions are considered, and the spin-independent cross section from radiative corrections is a few orders of magnitude smaller than the tree-level contributions.

KEYwords: Beyond Standard Model, Cosmology of Theories beyond the SM

ARXIV EPRINT: 1904.09785 


\section{Contents}

1 Introduction 1

2 The model 2

3 Effective operators 3

4 Results 5

5 Conclusion $\quad 9$

$\begin{array}{ll}\text { A Integrations } & 9\end{array}$

$\begin{array}{ll}\text { B Nuclear form factor } & 10\end{array}$

\section{Introduction}

Various observations have confirmed the existence of dark matter in our universe, whose relic density, derived by measuring the cosmic microwave background, large scale structure and galaxy formation, is about $0.1198 \pm 0.0033$ [1]. The standard model (SM) of particle physics contains no cold dark matter candidate, and the nature of dark matter remains elusive. There are many dark matter candidates with masses ranging from $10^{-20} \mathrm{eV}$ to $10^{55} \mathrm{GeV}$, of which the weakly interacting massive particle (WIMP) [2-7] is well-motivated as it can naturally explain the observed relic density via the thermal freeze-out with its mass at the electroweak scale and with weak couplings to the SM particles.

There are three (direct or indirect) ways of detecting WIMPs in laboratories: looking for the scattering between WIMPs and nucleon in underground laboratories by measuring the nuclear recoil energy in the kilo-electronvolt scale, detecting the flux of cosmic rays injected by the WIMP annihilations or decays with the help of satellites or telescopes, and producing WIMPs at the Large Hardron Collider (LHC) where the signal of WIMP is missing transverse momentum or missing energy. Of these three efforts, the first detection method is most straightforward since the astrophysical sources of cosmic rays have not been clearly determined in indirect detection experiments, and LHC is actually a mediator machine in dark matter detections.

Benefiting from technological advances, direct detection experiments such as LUX [8], PandaX-II [9] and XENON1T [10] have made tremendous strides in increasing precision and detecting efficiency. In an ideal status, one can detecting arbitrarily small direct detection cross section by continuously increasing the exposure, however it is well-known that direct detection experiments will soon reach to an irreducible background from coherent elastic neutrino-nuclei scattering, the so-called "neutrino floor" [11]. The current direct detection 
techniques will not be able to distinguish the signal of dark matter from that of neutrinos if the signal lies below the neutrino floor. That is to say the neutrino floor is the border of new and "old" direct detection techniques [12]. As a result, the precision calculation of the direct detection cross sections will be important whenever one wants to examine as many dark matter models as possible with the help of current direct detection techniques.

In this paper, we study the direct detections (DD) of a vector portal Majorana dark matter (MDM) [13]. The vector mediator model is one of the simplest dark matter models, whose phenomenology has been widely studied in refs. [14-24]. In this model $\chi$ is a Majorana dark matter, $V_{\mu}$ is a vector mediator, whose mass may arise from the spontaneous breaking of certain $\mathrm{U}(1)$ gauge symmetry, and $V_{\mu}$ may couple to the SM via a vector current or axial-vector current. In some models the vector portal is in associated with the Higgs portal since the scalar that causes the spontaneous breaking of the new $U(1)$ gauge symmetry may mix with the SM Higgs. Here we assume the mixing is negligibly small, thus the scattering of $\chi$ off the nuclei is only mediated by the $V_{\mu}$. The direct detection cross section $\sigma$ is either spin-independent but suppressed by the dark matter velocity or spin-dependent $[25,26]$. As a result, the spin-independent cross section $\sigma_{S I}$, generated at the loop level [27-41], turns out to be important as it may be still possible to examine these models with the current DD technique if $\sigma_{S I}$ arising from radiative corrections lies above the neutrino floor. We calculate effective operators for the evaluation of MDM-nucleon spin independent scattering cross section following [42, 43]. The WIMP-gluon effective operator $[44,45]$ raising at the two-loop level is also derived. Numerical simulations show that the $\sigma_{S I}$ from radiative corrections is reachable by the current DD technique for light MDM. Considering the constraint of pertubativty, radiative corrections are several orders of magnitude smaller than the tree level contributions.

The remaining of the paper is organized as follows: in section 2 we give a brief introduction to the vector portal MDM model. Section 3 is focused on the calculation of Wilson coefficients of effective operators. Numerical results are presented in section 4 and the last part is concluding remarks. Expressions of loop functions are listed in the appendix A. Nuclear form factors are given in the appendix B.

\section{The model}

In this section, we review the vector portal dark matter model. The simplified model contains a Majorana fermion $\chi$ and a new vector boson $V_{\mu}$ in addition to the SM particles. The Lagrangian for $\chi$ can be written as ${ }^{1}$

$$
\mathcal{L}_{\chi}=\frac{1}{2} \bar{\chi} i \not \partial \chi+\frac{1}{2} g_{V} \bar{\chi} \gamma^{\mu} \gamma^{5} V_{\mu} \chi-\frac{1}{2} m_{\chi} \bar{\chi} \chi
$$

where $m_{\chi}$ is the dark matter mass, $g_{V}$ is the new gauge coupling. The ultraviolet completed model contains a $\mathrm{U}(1)$ gauge symmetry and a complex scalar $\Phi\left(\equiv \frac{1}{\sqrt{2}}\left(\phi+i G+v_{\Phi}\right)\right)$ which is charged under the new $\mathrm{U}(1)$ and whose vacuum expectation value (VEV) $v_{\Phi}$ leads to

\footnotetext{
${ }^{1}$ The conventional kinematic term can be written as $\overline{\chi_{P}} i \not D \chi_{P}$, where $P$ is the chirality projection operator. Considering $\chi=\chi_{P}+\chi_{P}^{C}$, one arrives at the Lagrangian in eq. (2.1).
} 
the spontaneous breaking of the new gauge symmetry as well as the origin of masses of $\chi$ and $V$. In this case there will be a new Yukawa interaction, $\frac{1}{2} y_{\chi} \overline{\chi_{P}^{C}} \Phi \chi_{P}+$ h.c., where the subindex $P$ represents the chirality, and eq. (2.1) needs to extended with the $\frac{1}{2} \bar{\chi} \phi \chi$ term. In the case where $\phi$ mixies with the SM Higgs, there will be Higgs portal interactions. Notice that the mixing is caused by the quartic term: $\lambda_{H \Phi} \Phi^{\dagger} \Phi H^{\dagger} H . \lambda_{H \Phi}$ should be small due to constraints from precision observables as well as Higgs measurements at the LHC. Here we assume the mixing is negligible.

For interactions of new gauge boson with the SM particles, $V_{\mu}$ may couple to vector bilinears or axial-vector bilinears, or both, depending on the U(1) charge settings of the SM fermions. For example, new gauge interactions are $g_{V} \bar{f} \gamma^{\mu} f$ in $\mathrm{U}(1)_{B-L}[46], \mathrm{U}(1)_{B+L}[47]$ and $\mathrm{U}(1)_{B_{i}-L_{j}}$ models. While in the model where two chiral components carry opposite $\mathrm{U}(1)$ charges, new gauge interactions will be $g_{V} \bar{f} \gamma^{\mu} \gamma^{5} f$. If only one certain chirality component carries non-zero charge, new gauge interactions will be $g_{V} \bar{f} \gamma^{\mu} P_{A} V_{\mu} f$ [48], where $P_{A}=P_{L}$ or $P_{R}$. In this paper we carry out model independent study and investigate the DD cross section of vector current and axial-vector current scenarios separately. Interactions of $V_{\mu}$ with quarks are then

$$
\begin{array}{ccc}
\mathcal{L}_{I}^{q} \in g_{q} \bar{q} \gamma^{\mu} q V_{\mu}, & & (\text { scenario A) } \\
\mathcal{L}_{I I}^{q} \in g_{q} \bar{q} \gamma^{\mu} \gamma^{5} q V_{\mu}, & & \text { (scenario B) }
\end{array}
$$

where $g_{q}=\zeta g_{V}$ with $\zeta$ the $\mathrm{U}(1)$ hyper-charge of the quark $q$. Free parameters in these models are thus $m_{\chi}, m_{V}, g_{V}$ and $\zeta$.

\section{$3 \quad$ Effective operators}

In this section we calculate effective operators relevant for the spin-independent scattering cross section of $\chi$ with a nucleon. Following refs. [42, 43], we write down the effective $\chi$-quark interactions in terms of the higher-dimensional operators:

$$
\mathcal{L}_{\text {eff }}=\kappa_{0} \bar{\chi} \gamma^{\mu} \gamma^{5} \chi \bar{q} \Gamma q+\sum_{p=q, g} \frac{1}{2} \kappa_{1 p} \bar{\chi} \chi \mathcal{O}_{s}^{p}+\frac{1}{2} \kappa_{2 q} \bar{\chi} i \partial^{\mu} \gamma^{\nu} \chi \mathcal{O}_{\mu \nu}^{q}+\frac{1}{2} \kappa_{3 q} \bar{\chi} i \partial^{\mu} i \partial^{\nu} \chi \mathcal{O}_{\mu \nu}^{q},
$$

where $\Gamma=\gamma^{\mu}$ for scenario A and $\Gamma=\gamma^{\mu} \gamma^{5}$ for scenario B, $\kappa_{i p}$ are wilson coefficients, $\mathcal{O}_{s}^{q}=m \bar{q} q, \mathcal{O}_{s}^{g}=-\frac{9}{8} \alpha_{s} G^{A \mu \nu} G_{\mu \nu}^{A}$ and $\mathcal{O}_{\mu \nu}^{q}$ is the twist-2 operator, defined by

$$
\mathcal{O}_{\mu \nu}^{q}=\frac{1}{2} \bar{q}\left(\partial_{\mu} \gamma_{\nu}+\partial_{\nu} \gamma_{\mu}-\frac{1}{2} g_{\mu \nu} \not \partial\right) q .
$$

These effective operators are defined at the mass scale of $V_{\mu}$, which is assumed to be heavier than all the SM particles. In the following, we calculate Wilson coefficients at the leading and the next-to-leading order.

The leading order contribution to the Wilson coefficient $\kappa_{0}$ arises from the tree level diagram by exchanging the vector boson $V_{\mu}$,

$$
\kappa_{0}=\frac{\zeta g_{V}^{2}}{2 m_{V}^{2}}
$$

where $m_{V}$ is the mass of $V_{\mu}$. 

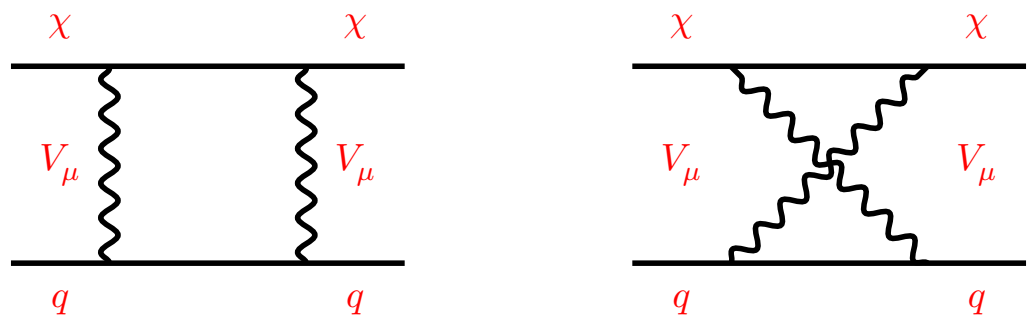

Figure 1. Box diagrams for the effective quark-WIMP interactions.

$\kappa_{1,2,3}^{q}$ arise from the box diagrams given in the figure 1 . We calculate the diagrams in the zero-momentum transfer limit, and expand the amplitude in term of the quark momentum, which is non-relativistic, then we decompose results into effective operators following ref. [38]. For the scenario A, the relevant Wilson coefficients are

$$
\begin{aligned}
\kappa_{A 1 q}= & -\frac{m_{\chi}}{2} \alpha_{V}^{2}\left\{3 X_{2}\left(m_{\chi}^{2}, m_{V}^{2}, 0, m_{\chi}^{2}\right)+Y_{2}\left(m_{\chi}^{2}, m_{V}^{2}, 0, m_{\chi}^{2}\right)+12 Z_{001}\left(m_{\chi}^{2}, m_{V}^{2}, m_{\chi}^{2}\right)\right. \\
& \left.+6 Z_{00}\left(m_{\chi}^{2}, m_{V}^{2}, m_{\chi}^{2}\right)+m_{\chi}^{2}\left[3 Z_{11}\left(m_{\chi}^{2}, m_{V}^{2}, m_{\chi}^{2}\right)+2 Z_{111}\left(m_{\chi}^{2}, m_{V}^{2}, m_{\chi}^{2}\right)\right]\right\} \\
\kappa_{A 2 q}= & -2 \alpha_{V}^{2}\left\{2 Z_{00}\left(m_{\chi}^{2}, m_{V}^{2}, m_{\chi}^{2}\right)+8 Z_{001}\left(m_{\chi}^{2}, m_{V}^{2}, m_{\chi}^{2}\right)+m_{\chi}^{2}\left[Z_{11}\left(m_{\chi}^{2}, m_{V}^{2}, m_{\chi}^{2}\right)\right.\right. \\
& \left.\left.+Z_{111}\left(m_{\chi}^{2}, m_{V}^{2}, m_{\chi}^{2}\right)\right]-X_{2}\left(m_{\chi}^{2}, m_{V}^{2}, 0, m_{\chi}^{2}\right)-Y_{2}\left(m_{\chi}^{2}, m_{V}^{2}, 0, m_{\chi}^{2}\right)\right\} \\
\kappa_{A 3 q}= & -2 \alpha_{V}^{2}\left[2 Z_{11}\left(m_{\chi}^{2}, m_{V}^{2}, m_{\chi}^{2}\right)+Z_{111}\left(m_{\chi}^{2}, m_{V}^{2}, m_{\chi}^{2}\right)\right]
\end{aligned}
$$

where $\alpha_{V}=g_{V}^{2} / 4 \pi$. The definitions of the loop functions and their explicit expressions are given in the appendix A. Loop functions are evaluated with the help of the Package-X [51, 52].

For scenario B the Wilson coefficients are

$$
\begin{aligned}
\kappa_{B 1 q}= & +\frac{m_{\chi}}{2} \alpha_{V}^{2}\left\{9 X_{2}\left(m_{\chi}^{2}, m_{V}^{2}, 0, m_{\chi}^{2}\right)+3 Y_{2}\left(m_{\chi}^{2}, m_{V}^{2}, 0, m_{\chi}^{2}\right)-6 Z_{00}\left(m_{\chi}^{2}, m_{V}^{2}, m_{\chi}^{2}\right)\right. \\
& \left.+13 Z_{001}\left(m_{\chi}^{2}, m_{V}^{2}, m_{\chi}^{2}\right)-m_{\chi}^{2}\left[3 Z_{11}\left(m_{\chi}^{2}, m_{V}^{2}, m_{\chi}^{2}\right)-2 Z_{111}\left(m_{\chi}^{2}, m_{V}^{2}, m_{\chi}^{2}\right)\right]\right\}, \\
\kappa_{B 2 q}= & -2 \alpha_{V}^{2}\left\{2 Z_{00}\left(m_{\chi}^{2}, m_{V}^{2}, m_{\chi}^{2}\right)-8 Z_{001}\left(m_{\chi}^{2}, m_{V}^{2}, m_{\chi}^{2}\right)+m_{\chi}^{2}\left[Z_{11}\left(m_{\chi}^{2}, m_{V}^{2}, m_{\chi}^{2}\right)\right.\right. \\
& \left.\left.-Z_{111}\left(m_{\chi}^{2}, m_{V}^{2}, m_{\chi}^{2}\right)\right]-X_{2}\left(m_{\chi}^{2}, m_{V}^{2}, 0, m_{\chi}^{2}\right)-Y_{2}\left(m_{\chi}^{2}, m_{V}^{2}, 0, m_{\chi}^{2}\right)\right\}, \\
\kappa_{B 3 q}= & -2 \alpha_{V}^{2}\left[2 Z_{11}\left(m_{\chi}^{2}, m_{V}^{2}, m_{\chi}^{2}\right)-Z_{111}\left(m_{\chi}^{2}, m_{V}^{2}, m_{\chi}^{2}\right)\right] .
\end{aligned}
$$

The effective MDM-gluon interactions arise at the two-loop level. Relevant Feynman diagrams are given in the figure 2. In this paper we only take into account the effect of twist-0 MDM-gluon operator and neglecting the contribution of higher twist MDMgluon operators. One loop correction to the two-point function of gauge boson in the gluon background field has been calculated in ref. [49] by taking the Fock-Schwinger gauge [53, 54] for the gluon field, which, mapped into our cases, can be written as

$$
i \Pi_{V V}^{(f) \alpha \beta}=-\frac{1}{3} \frac{i \zeta^{2} g_{s}^{2}}{16 \pi} G_{\mu \nu}^{a} G^{a \mu \nu}\left(\frac{g_{V}^{2}}{q^{2}} g^{\alpha \beta}-\frac{g_{V}^{2}}{q^{4}} q^{\alpha} q^{\beta}\right)
$$

where $g_{s}$ is the coupling of the strong interaction, $f$ indicates the flavor running in the fermion loop, $q$ is the momentum of gauge boson. Notice that eq. (3.10) is universal for both the scenario A and the scenario B. 

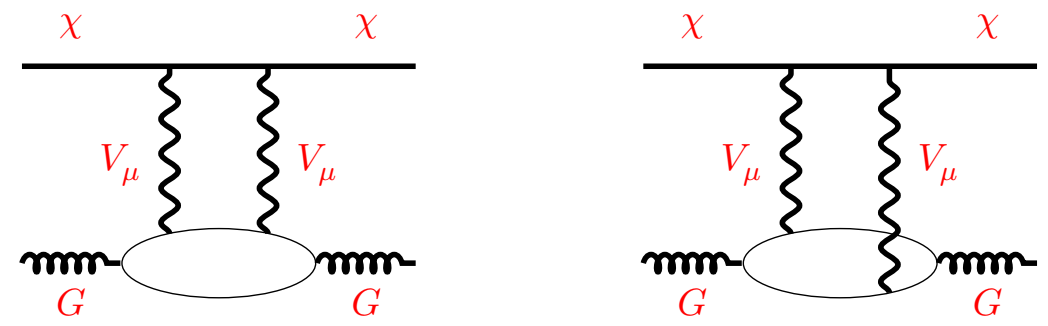

Figure 2. Two-loop Feynman diagrams for the effective gluon-WIMP interactions.

With the help of eq. (3.10), one can write down the Wilson coefficient of the effective $\chi$-gluon operator, which takes the form

$$
\begin{aligned}
\kappa_{A g(B g)}= & \frac{\alpha_{V}^{2}}{54} \zeta^{2} n_{f} m_{\chi}\left(6 X_{2}\left(m_{\chi}^{2}, m_{V}^{2}, 0, m_{\chi}^{2}\right)+2 Y_{2}\left(m_{\chi}^{2}, m_{V}^{2}, 0, m_{\chi}^{2}\right)\right. \\
& \left.+6 Z_{001}\left(m_{\chi}^{2}, m_{V}^{2}, m_{\chi}^{2}\right)-6 Z_{00}\left(m_{\chi}^{2}, m_{V}^{2}, m_{\chi}^{2}\right)+m_{\chi}^{2} Z_{11}\left(m_{\chi}^{2}, m_{V}^{2}, m_{\chi}^{2}\right)\right)
\end{aligned}
$$

where $n_{f}$ is the number of quarks that carry nonzero $\mathrm{U}(1)$ charge.

Wilson coefficients given above are matched to the simplified model at the scale $\mu \approx m_{V}$. The energy scale for the DM direct detections is about the nuclear energy scale. It has been shown in ref. [29] that effects from the running of renormalization group equations might be sizable in certain vector-portal scenario. We use the public code RUNDM [29] to evolve the running of $\kappa_{0}$ and evaluate running effects of other Wilson coefficients following refs. $[45,55-58]$.

\section{Results}

In this section we present results for the MDM-nucleon scattering cross section. We start by determining the $g_{V}$ using the observed relic density. As was mentioned in the previous section, there are four parameters in the vector portal, which are all relevant for both the relic abundance and the direct detection cross section. The thermal relic abundance is determined by the following processes: $\bar{\chi} \chi \rightarrow \bar{f} f$ and $\bar{\chi} \chi \rightarrow V_{\mu} V^{\mu}$, where $f$ indicates the SM fermion. The first channel depends on the parameter $\zeta$, while the second channel does not. For $m_{\chi}<m_{V}$, the channel $\bar{\chi} \chi \rightarrow V_{\mu} V^{\mu}$ is kinematically forbidden, and thus the combination $\zeta g_{V}^{2}$ can be determined in this mass range.

We show in the figure 3 , the new gauge coupling $g_{V}$ as the function of the dark matter mass $m_{\chi}$ determined by the observed relic abundance $\Omega h^{2}=0.1198$, by setting $m_{V}=3 \mathrm{TeV}$ and $\zeta=1$. The solid and dashed lines are the cases of scenario A and scenario $\mathrm{B}$, respectively. The first dip of the plot appears at $m_{\chi} \sim m_{V} / 2$, where the annihilation $\bar{\chi} \chi \rightarrow \bar{f} f$ is resonantly enhanced. The second dip of the plot at $m_{\chi} \sim m_{V}$ is due to the opening of the annihilation channel $\bar{\chi} \chi \rightarrow V_{\mu} V^{\mu}$. Notice that $g_{V}$ becomes almost scenario independent as $m_{\chi} \sim m_{V}$. We show in the right panel of the figure 3 contours of $g_{V}$ in the $m_{\chi}-m_{V}$ plane by setting $\zeta=1$. Notice that $\mathcal{O}\left(g_{V}\right) \sim 1$ for $m_{V} \sim m_{\chi} \sim \mathcal{O}(1) \mathrm{TeV}$.

We show in the figure 4 Wilson coefficients as the function of the dark matter mass $m_{\chi}$ by setting $g_{V}=\zeta=1$ and $m_{V}=1 \mathrm{TeV}$. The plots in the left-panel and right-panel 

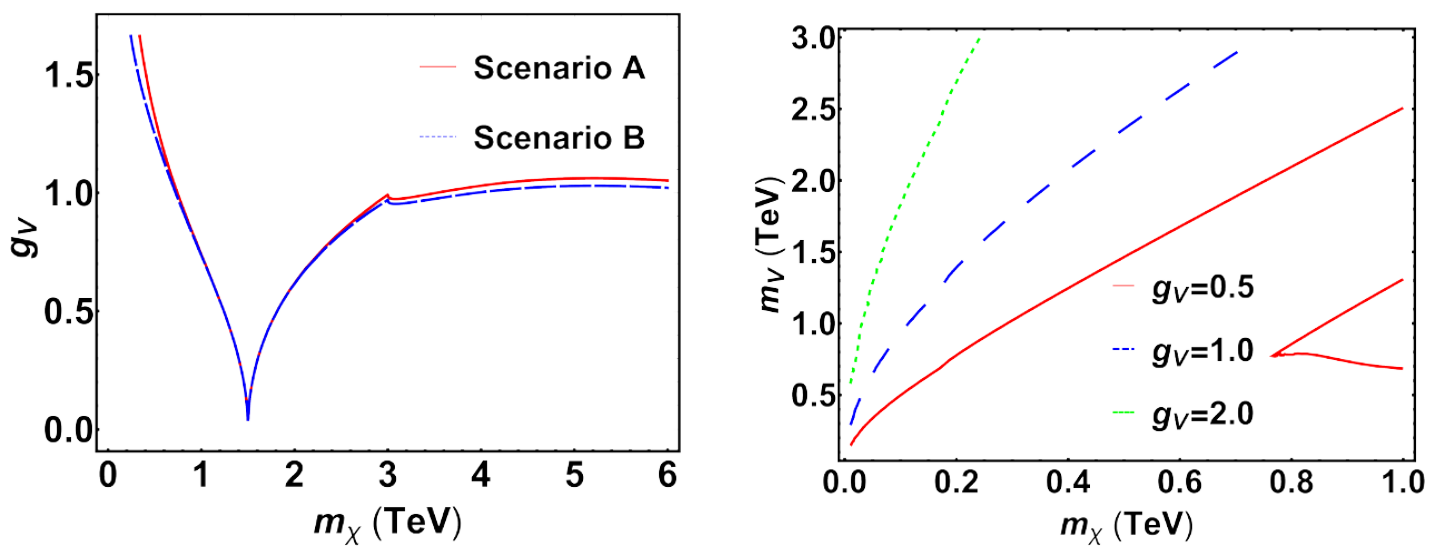

Figure 3. Left panel: new gauge coupling as the function of the dark matter mass by setting $m_{V}=3 \mathrm{TeV}$ and $\zeta=1$, constrained by the observed relic density. The solid and dashed lines correspond to the scenario A and scenario B, respectively. Right panel: contours of $g_{V}$ in the $m_{\chi}-m_{V}$ plane by setting $\zeta=1$.
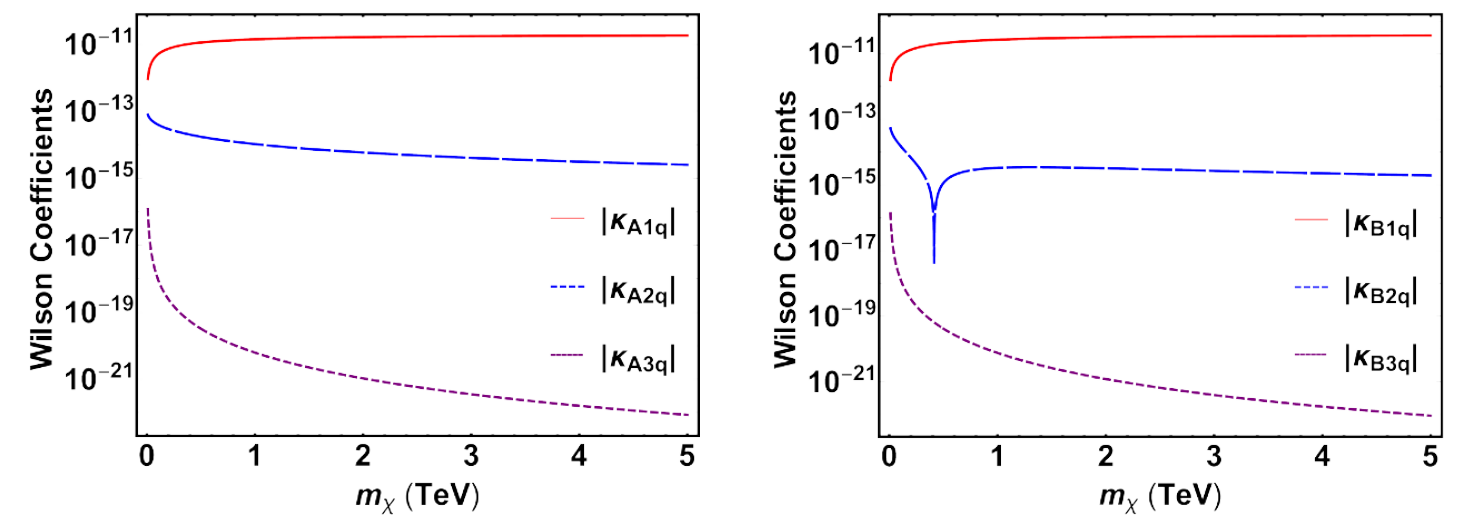

Figure 4. Wilson coefficients as the function of the dark matter mass by setting $\zeta=g_{V}=1$ and $m_{V}=1 \mathrm{TeV}$ for scenario A (left-panel) and scenario B (right-panel).

correspond to cases of scenario A and B, respectively. As can be seen, the Wilson coefficient of the scalar type interaction is largest and is comparable to that in pseudo-scalar portal model [38]. Wilson coefficients in scenario B is similar to these in scenario A except $\kappa_{B 2 q}$ at $m_{\chi} \sim m_{V}$, which is due to the cancellation of various contributions. The Wilson coefficient $\kappa_{g}$ is of the order $\mathcal{O}\left(10^{-11}\right)$ by setting $g_{V}=\zeta=1$.

Now we calculate the MDM-nucleon scattering cross section. For scenario A, there is velocity suppressed spin-independent scattering cross section at the leading order,

$$
\sigma_{\mathrm{SI}}^{\mathrm{LO}} \approx \frac{36 \pi \zeta^{2} \alpha_{V}^{2} \mu^{2} v^{2}}{m_{V}^{4}},
$$

where $\mu$ is the reduced mass of $\chi$ and nucleon system, $v$ is the velocity of the dark matter. Effective interactions given in the eq. (3.1) contribute to the scattering cross section at the next-to-leading order,

$$
\sigma_{\mathrm{SI}}^{\mathrm{NLO}}=\frac{\mu^{2} m_{N}^{2}}{\pi}\left[\sum_{q} \kappa_{A 1 q} f_{T q}^{N}+\kappa_{g} f_{T_{g}}^{N}+\frac{3}{4} \sum_{q}\left(m_{\chi} \kappa_{A 2 q}+m_{\chi}^{2} \kappa_{A 3 q}\right)\left(q^{N}(2)+\bar{q}^{N}(2)\right)\right]^{2}
$$




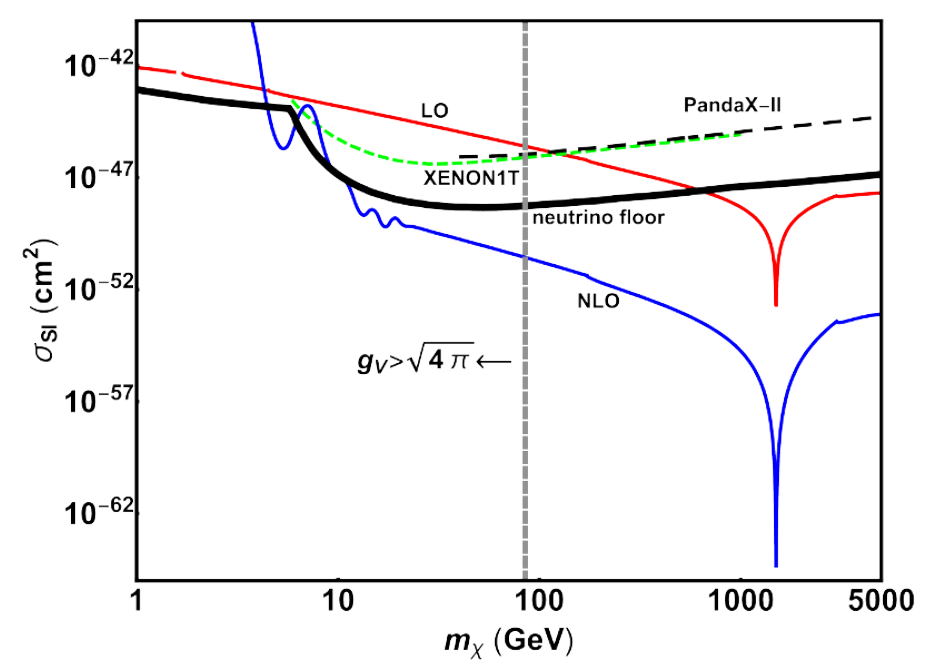

Figure 5. Direct detection cross section as the function of the dark matter mass by setting $\zeta=1$ and $m_{V}=3 \mathrm{TeV}$ for scenario A. The red and blue solid lines correspond to the LO and NLO contributions, respectively. The black dashed and green dashed lines are separately constraints of Pandax-II and XENON1T. The black solid line is the so-called neutrino floor. The gray vertical dashed line is the constraint of perturbativity.

where $N$ stands for $(p, n)$ with $m_{N}$ its mass, $f_{T_{q}}^{N}$ is the quark matrix element defined by $\left\langle N\left|m_{q} \bar{q} q\right| N\right\rangle=m_{N} f_{T_{q}}^{N}, f_{T g}^{N}$ is the gluon matrix element defined by $-\frac{9 \alpha_{s}}{8 \pi}\left\langle N\left|G_{\mu \nu}^{a} G^{a \mu \nu}\right| N\right\rangle=$ $m_{N} f_{T_{g}}^{N}, q^{N}(2)$ and $\bar{q}^{N}(2)$ are second moments for quark distribution functions of $N$. Numerical values of $f_{T_{q}}^{N}, f_{T_{g}}^{N}$ as well as $q^{N}(2)$ and $\bar{q}^{N}(2)$ are listed in the appendix B. Notice that there is no interference between the leading oder and the next to leading oder contributions. As a result, the total cross section for scenario $\mathrm{A}$ can be written as $\sigma_{\mathrm{SI}}^{\mathrm{tot}}=\sigma_{\mathrm{SI}}^{\mathrm{LO}}+\sigma_{\mathrm{SI}}^{\mathrm{NLO}}$.

For scenario $\mathrm{B}$, the $\chi$-nucleon scattering cross section is spin-dependent at the leading order,

$$
\sigma_{\mathrm{SD}}^{\mathrm{LO}}=64 \pi \zeta^{2} \alpha_{V}^{2} \frac{\mu^{2}}{m_{V}^{4}}\left(\sum_{q} \Delta_{q}^{N}\right)^{2} J_{N}\left(J_{N}+1\right)
$$

where $\Delta_{q}^{N}$ is the spin fraction of quark $q$, defined by $2 \Delta_{q}^{N} s^{\mu}=\left\langle N\left|\bar{q} \gamma^{\mu} \gamma^{5}\right| N\right\rangle$ with $s^{\mu}$ the nucleon spin four-vector, $J_{N}$ is the angular momentum of the nucleon. $\Delta_{q}^{N}$ are measured in DIS and one has $\Delta_{u}^{p}=0.77, \Delta_{d}^{p}=-0.47$ and $\Delta_{s}^{p}=-0.15$ [50]. The $\chi$-nucleon scattering cross section at the next-to-leading order can be spin independent, whose expression is the same as eq. (4.2), up to following replacements, $\kappa_{A i q} \rightarrow \kappa_{B i q}(i=1,2,3)$.

As an illustration, we show in the figure 5 the direct detection cross section as the function of the dark matter mass $m_{\chi}$ for scenario A, by setting $m_{V}=3 \mathrm{TeV}$ and $\zeta=1$. The red solid and blue dashed lines correspond to $\sigma_{\mathrm{SI}}^{\mathrm{LO}}$ and $\sigma_{\mathrm{SI}}^{\mathrm{NLO}}$, respectively. The black dashed and green dashed lines are separately constraints given by the PandaX-II [9] and XENON1T [8] experiments. One can conclude from the plot that even though the $\sigma_{\mathrm{SI}}^{\mathrm{LO}}$ is suppressed by the dark matter velocity it is still sizable and the current constraints have excluded the low dark matter mass region $\left(6 \mathrm{GeV}<m_{\chi}<120 \mathrm{GeV}\right)$. The next-to-leading contribution is reachable by the current DD technique for very light MDM, and it is about 

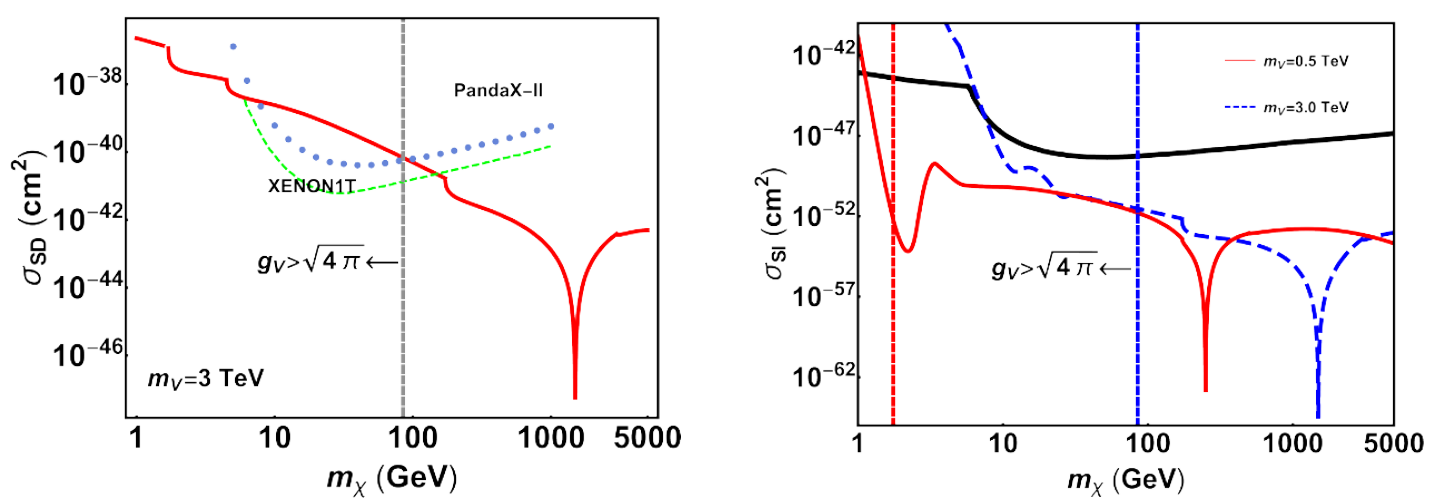

Figure 6. Left-panel: spin-dependent $\chi$-neutron scattering cross section as the function of the dark matter mass by setting $\zeta=1$ and $m_{V}=3 \mathrm{TeV}$ for scenario B. The green and blue dotted lines are constraints of XENON1T and PandaX-II, respectively. Right-panel: spin-independent $\chi$-neutron scattering cross section as the function of $m_{\chi}$ by setting $\zeta=1$ and $m_{V}=3 \mathrm{TeV}$ for scenario B. The black solid line is the neutrino floor.

5 orders of magnitude smaller than the LO contribution for heavy MDM. The reason that $\sigma^{\mathrm{NLO}}$ being sensitive to light MDM is that $\sigma^{\mathrm{NLO}}$ is proportional to $g_{V}^{8}$ while $\sigma^{\mathrm{LO}}$ is proportional $g_{V}^{4}$. As a result, a slight increase of $g_{V}$ may enhance the $\sigma^{\mathrm{NLO}}$ obviously. The vertical dashed line in the plot is the constraint of perturbativity [64, 65], to the left of which one has $g_{V}>\sqrt{4 \pi}$, that is excluded by the perturbative unitarity.

We show in the left-panel of the figure 6 the spin-dependent $\chi$-neutron scattering cross section as the function of $m_{\chi}$ for scenario B, by setting $\zeta=1$ and $m_{V}=3 \mathrm{TeV}$. The blue and green dotted lines are constraints of PandaX-II [59] and XENON1T [60] experiments, respectively. One can see that the mass region $(8,90) \mathrm{GeV}$ is already excluded. The vertical dashed line is the constraint of perturbative unitarity. We show in the right-panel of the figure 6 the spin-independent cross section as the function of $m_{\chi}$ for scenario B. The solid and dashed lines correspond to $m_{V}=3 \mathrm{TeV}$ and $0.5 \mathrm{TeV}$, respectively. The black solid line is the neutrino floor. It is clear that the spin-independent cross section is too small to be detected in the near future for heavy MDM. Vertical lines are constraints of perturbativity, where the blue and red vertical lines correspond to $m_{V}=3 \mathrm{TeV}$ and $0.5 \mathrm{TeV}$, respectively.

Notice that we did not consider constraints of LHC [23, 63, 64] since we are only focusing on direct detections in underground laboratories, however constraints from LHC are definitely important when considering a specific vector portal MDM model. Direct search for the spin-1 mediator, especially the search for dijet resonances at the LHC, may put strong constraint on various vector portal models. After producing dijet events in a Monte Carlo generator, applying the cuts, and comparing the predicted dijet invariant mass distributions with the experimental results, one may get limits on a $Z^{\prime}$ model. The resulting largest value of $g_{q}$ (at the $95 \%$ confidence level) depends on $m_{V}$ and $\Gamma_{V}$, and takes the form [23]

$$
g_{q, \max }^{4}=a\left(m_{V}\right)\left(\frac{\Gamma_{V}}{m_{V}}\right)^{b\left(m_{V}\right)}+c\left(m_{V}\right)
$$

where $a(3000 \mathrm{GeV})=0.30428, b(3000 \mathrm{GeV})=1.315$ and $c(3000 \mathrm{GeV})=7.375 \times 10^{-4}[23]$. 
To constrain the dark matter coupling using the dijet results, one may first reconstruct the dark matter coupling using the observed relic abundance, which is the function of $g_{p}, m_{V}$ and $m_{\chi}$. Then we get its lower bound from the combination of the observed relic density and LHC dijet results. Since the decay width $\Gamma_{V}$ is model dependent (there might be invisible decays of heavy vector boson) and eq. (4.4) is valid for $\Gamma_{V} / m_{V} \leq 0.3$, we will not apply these constraints to our plots.

\section{Conclusion}

In this paper we have studied the direct detection cross section of Majorana dark matter $\chi$ in the vector portal. At the leading order, the cross section is either velocity suppressed or spin-dependent, and current constraints given by XENON1T and PandaX-II experiments can only rule out a narrow mass range depending on the inputs. Future direct detection experiments may improve the detection sensitivity to higher level. Next-to-leading order corrections may turn out to be important. We have derived the effective $\chi$-quark interactions at the one-loop level and the effective $\chi$-gluon interaction at the two-loop level. We got the full formula of SI direct detection cross section at the next-to-leading order. Our numerical results show that the next-to-leading order corrections to the SI cross section is several orders of magnitude smaller than the leading order contribution for the heavy mediator case.

\section{Acknowledgments}

This work was supported by the National Natural Science Foundation of China under grant No. 11775025 and the Fundamental Research Funds for the Central Universities under grant No. 2017NT17.

\section{A Integrations}

We list in this appendix definitions of integration used in this paper, given by [49]:

$$
\begin{aligned}
\int \frac{d^{4} k}{(2 \pi)^{4}} \frac{1}{\left[(p+k)^{2}-M^{2}\right] k^{2}\left[k^{2}-m^{2}\right]^{2}}= & \frac{i}{16 \pi^{2}} X_{2}\left(p^{2}, M^{2}, 0, m^{2}\right) \\
\int \frac{d^{4} k}{(2 \pi)^{4}} \frac{k_{\mu}}{\left[(p+k)^{2}-M^{2}\right] k^{2}\left[k^{2}-m^{2}\right]^{2}}= & \frac{i}{16 \pi^{2}} p_{\mu} Y_{2}\left(p^{2}, M^{2}, 0, m^{2}\right) \\
\int \frac{d^{4} k}{(2 \pi)^{4}} \frac{k_{\mu} k_{\nu}}{\left[(p+k)^{2}-M^{2}\right] k^{4}\left[k^{2}-m^{2}\right]^{2}}= & \frac{i}{16 \pi^{2}}\left[p_{\mu} p_{\nu} Z_{11}\left(p^{2}, M^{2}, m^{2}\right)+g_{\mu \nu} Z_{00}\left(p^{2}, M^{2}, m^{2}\right)\right] \\
\int \frac{d^{4} k}{(2 \pi)^{4}} \frac{k_{\mu} k_{\nu} k_{\sigma}}{\left[(p+k)^{2}-M^{2}\right] k^{4}\left[k^{2}-m^{2}\right]^{2}}= & \frac{i}{16 \pi^{2}}\left[p_{\mu} p_{\nu} p_{\sigma} Z_{111}\left(p^{2}, M^{2}, m^{2}\right)\right. \\
& \left.+\left(g_{\mu \nu} p_{\sigma}+g_{\mu \sigma} p_{\nu}+g_{\nu \sigma} p_{\mu}\right) Z_{000}\left(p^{2}, M^{2}, m^{2}\right)\right]
\end{aligned}
$$

These integrations are evaluated using package-X [51, 52]. 


\section{B Nuclear form factor}

To calculate the WIMP-nucleon scattering cross section, one needs following nuclear form factors: $\left\langle N\left|m_{q} \bar{q} q\right| N\right\rangle=m_{N} f_{T_{q}}^{N},(q=u, d, s),\left\langle N\left|-\frac{9 \alpha_{s}}{8 \pi} G_{\mu \nu}^{a} G^{a \mu \nu}\right| N\right\rangle=m_{N} f_{T_{g}}^{N},\left\langle N\left|\mathcal{O}_{\mu \nu}^{q}\right| N\right\rangle=$ $\frac{1}{m_{N}}\left(p_{\mu}^{N} p_{\nu}^{N}-\frac{1}{4} m_{N}^{2} g_{\mu \nu}\right)\left(q^{N}(2)+\bar{q}^{N}(2)\right)$, where $m_{N}$ is nucleon mass, $f_{T_{q}}^{N}$ and $f_{T_{g}}^{N}$ are form factors taken from micrOmegas [61], $q^{N}(2)$ and $\bar{q}^{N}(2)$ are the second momentum for quark distribution functions evaluated at $\mu=m_{Z}$ by using CTEQ PDF [62]. Specific inputs are [61]

$$
\begin{aligned}
& f_{T_{u}}^{p}=0.0153, \quad f_{T_{d}}^{p}=0.0191, \quad f_{T_{s}}^{p}=0.0447, \\
& f_{T_{u}}^{n}=0.0110, \quad f_{T_{d}}^{n}=0.0273, \quad f_{T_{s}}^{n}=0.0447, \\
& f_{T_{g}}^{N}=1-\sum_{q=u, d, s} f_{T_{q}}^{N}, \text { and }[38,62] \\
& u^{p}(2)=0.220, \quad d^{p}(2)=0.110, \quad s^{p}(2)=0.026, \quad c^{p}(2)=0.019, \quad b^{p}(2)=0.012, \\
& \bar{u}^{p}(2)=0.034, \quad \bar{d}^{p}(2)=0.036, \quad \bar{s}^{p}(2)=0.026, \quad \bar{c}^{p}(2)=0.019, \quad \bar{b}^{p}(2)=0.012 .
\end{aligned}
$$

Open Access. This article is distributed under the terms of the Creative Commons Attribution License (CC-BY 4.0), which permits any use, distribution and reproduction in any medium, provided the original author(s) and source are credited.

\section{References}

[1] Planck collaboration, Planck 2018 results. VI. Cosmological parameters, arXiv: 1807.06209 [INSPIRE].

[2] H. Goldberg, Constraint on the Photino Mass from Cosmology, Phys. Rev. Lett. 50 (1983) 1419 [Erratum ibid. 103 (2009) 099905] [INSPIRE].

[3] J.R. Ellis, J.S. Hagelin, D.V. Nanopoulos, K.A. Olive and M. Srednicki, Supersymmetric Relics from the Big Bang, Nucl. Phys. B 238 (1984) 453 [inSPIRE].

[4] G. Jungman, M. Kamionkowski and K. Griest, Supersymmetric dark matter, Phys. Rept. 267 (1996) 195 [hep-ph/9506380] [INSPIRE].

[5] G. Servant and T.M.P. Tait, Is the lightest Kaluza-Klein particle a viable dark matter candidate?, Nucl. Phys. B 650 (2003) 391 [hep-ph/0206071] [INSPIRE].

[6] H.-C. Cheng, J.L. Feng and K.T. Matchev, Kaluza-Klein dark matter, Phys. Rev. Lett. 89 (2002) 211301 [hep-ph/0207125] [INSPIRE].

[7] G. Bertone, D. Hooper and J. Silk, Particle dark matter: Evidence, candidates and constraints, Phys. Rept. 405 (2005) 279 [hep-ph/0404175] [INSPIRE].

[8] LUX collaboration, First results from the LUX dark matter experiment at the Sanford Underground Research Facility, Phys. Rev. Lett. 112 (2014) 091303 [arXiv:1310.8214] [INSPIRE].

[9] PandaX-II collaboration, Dark Matter Results from First 98.7 Days of Data from the PandaX-II Experiment, Phys. Rev. Lett. 117 (2016) 121303 [arXiv:1607.07400] [InSPIRE].

[10] XENON collaboration, First Dark Matter Search Results from the XENON1T Experiment, Phys. Rev. Lett. 119 (2017) 181301 [arXiv:1705.06655] [INSPIRE]. 
[11] J. Billard, L. Strigari and E. Figueroa-Feliciano, Implication of neutrino backgrounds on the reach of next generation dark matter direct detection experiments, Phys. Rev. D 89 (2014) 023524 [arXiv: 1307.5458] [INSPIRE].

[12] W. Chao, J.-G. Jiang, X. Wang and X.-Y. Zhang, Direct Detections of Dark Matter in the Presence of Non-standard Neutrino Interactions, JCAP 08 (2019) 010 [arXiv: 1904.11214] [INSPIRE].

[13] E. Dudas, Y. Mambrini, S. Pokorski and A. Romagnoni, (In)visible Z-prime and dark matter, JHEP 08 (2009) 014 [arXiv: 0904.1745] [INSPIRE].

[14] H. An, X. Ji and L.-T. Wang, Light Dark Matter and $Z^{\prime}$ Dark Force at Colliders, JHEP 07 (2012) 182 [arXiv:1202.2894] [INSPIRE].

[15] M.T. Frandsen, F. Kahlhoefer, A. Preston, S. Sarkar and K. Schmidt-Hoberg, LHC and Tevatron Bounds on the Dark Matter Direct Detection Cross-Section for Vector Mediators, JHEP 07 (2012) 123 [arXiv: 1204.3839] [INSPIRE].

[16] H. Dreiner, D. Schmeier and J. Tattersall, Contact Interactions Probe Effective Dark Matter Models at the LHC, EPL 102 (2013) 51001 [arXiv:1303.3348] [INSPIRE].

[17] A. Alves, S. Profumo and F.S. Queiroz, The dark $Z^{\prime}$ portal: direct, indirect and collider searches, JHEP 04 (2014) 063 [arXiv:1312.5281] [INSPIRE].

[18] G. Arcadi, Y. Mambrini, M.H.G. Tytgat and B. Zaldivar, Invisible $Z^{\prime}$ and dark matter: LHC vs LUX constraints, JHEP 03 (2014) 134 [arXiv: 1401.0221] [INSPIRE].

[19] O. Lebedev and Y. Mambrini, Axial dark matter: The case for an invisible $Z^{\prime}$, Phys. Lett. B 734 (2014) 350 [arXiv: 1403.4837] [INSPIRE].

[20] N.F. Bell, Y. Cai, R.K. Leane and A.D. Medina, Leptophilic dark matter with $Z^{\prime}$ interactions, Phys. Rev. D 90 (2014) 035027 [arXiv:1407.3001] [InSPIRE].

[21] A. Alves, A. Berlin, S. Profumo and F.S. Queiroz, Dark Matter Complementarity and the $Z^{\prime}$ Portal, Phys. Rev. D 92 (2015) 083004 [arXiv:1501.03490] [InSPIRE].

[22] A. De Simone and T. Jacques, Simplified models vs. effective field theory approaches in dark matter searches, Eur. Phys. J. C 76 (2016) 367 [arXiv: 1603.08002] [inSPIRE].

[23] M. Fairbairn, J. Heal, F. Kahlhoefer and P. Tunney, Constraints on $Z^{\prime}$ models from LHC dijet searches and implications for dark matter, JHEP 09 (2016) 018 [arXiv:1605.07940] [INSPIRE].

[24] Y. Cui and F. D'Eramo, Surprises from complete vector portal theories: New insights into the dark sector and its interplay with Higgs physics, Phys. Rev. D 96 (2017) 095006 [arXiv: 1705.03897] [INSPIRE].

[25] A.L. Fitzpatrick, W. Haxton, E. Katz, N. Lubbers and Y. Xu, The Effective Field Theory of Dark Matter Direct Detection, JCAP 02 (2013) 004 [arXiv: 1203.3542] [INSPIRE].

[26] N. Anand, A.L. Fitzpatrick and W.C. Haxton, Weakly interacting massive particle-nucleus elastic scattering response, Phys. Rev. C 89 (2014) 065501 [arXiv:1308.6288] [InSPIRE].

[27] U. Haisch and F. Kahlhoefer, On the importance of loop-induced spin-independent interactions for dark matter direct detection, JCAP 04 (2013) 050 [arXiv:1302.4454] [INSPIRE].

[28] A. Crivellin and U. Haisch, Dark matter direct detection constraints from gauge bosons loops, Phys. Rev. D 90 (2014) 115011 [arXiv:1408.5046] [INSPIRE]. 
[29] F. D'Eramo, B.J. Kavanagh and P. Panci, You can hide but you have to run: direct detection with vector mediators, JHEP 08 (2016) 111 [arXiv:1605.04917] [INSPIRE].

[30] A. Crivellin, F. D'Eramo and M. Procura, New Constraints on Dark Matter Effective Theories from Standard Model Loops, Phys. Rev. Lett. 112 (2014) 191304 [arXiv: 1402.1173] [INSPIRE].

[31] F. Bishara, J. Brod, B. Grinstein and J. Zupan, Renormalization Group Effects in Dark Matter Interactions, arXiv:1809.03506 [INSPIRE].

[32] T. Li, Revisiting the direct detection of dark matter in simplified models, Phys. Lett. B 782 (2018) 497 [arXiv: 1804.02120] [INSPIRE].

[33] N.F. Bell, G. Busoni and I.W. Sanderson, Loop Effects in Direct Detection, JCAP 08 (2018) 017 [Erratum ibid. 01 (2019) E01] [arXiv: 1803.01574] [INSPIRE].

[34] J. Hisano, K. Ishiwata and N. Nagata, A complete calculation for direct detection of Wino dark matter, Phys. Lett. B 690 (2010) 311 [arXiv:1004.4090] [INSPIRE].

[35] J. Hisano, K. Ishiwata, N. Nagata and T. Takesako, Direct Detection of Electroweak-Interacting Dark Matter, JHEP 07 (2011) 005 [arXiv: 1104.0228] [INSPIRE].

[36] F. Ertas and F. Kahlhoefer, Loop-induced direct detection signatures from CP-violating scalar mediators, JHEP 06 (2019) 052 [arXiv:1902.11070] [INSPIRE].

[37] K. Ishiwata and T. Toma, Probing pseudo Nambu-Goldstone boson dark matter at loop level, JHEP 12 (2018) 089 [arXiv:1810.08139] [INSPIRE].

[38] T. Abe, M. Fujiwara and J. Hisano, Loop corrections to dark matter direct detection in a pseudoscalar mediator dark matter model, JHEP 02 (2019) 028 [arXiv:1810.01039] [INSPIRE].

[39] W. Chao, G.-J. Ding, X.-G. He and M. Ramsey-Musolf, Scalar Electroweak Multiplet Dark Matter, JHEP 08 (2019) 058 [arXiv: 1812.07829] [INSPIRE].

[40] K. Ghorbani and P.H. Ghorbani, Leading Loop Effects in Pseudoscalar-Higgs Portal Dark Matter, JHEP 05 (2019) 096 [arXiv: 1812.04092] [INSPIRE].

[41] T. Li and P. Wu, Simplified dark matter models with loop effects in direct detection and the constraints from indirect detection and collider search, Chin. Phys. C 43 (2019) 113102 [arXiv: 1904.03407] [INSPIRE].

[42] J. Hisano, R. Nagai and N. Nagata, Effective Theories for Dark Matter Nucleon Scattering, JHEP 05 (2015) 037 [arXiv: 1502.02244] [INSPIRE].

[43] J. Hisano, Effective theory approach to direct detection of dark matter, arXiv:1712.02947 [INSPIRE].

[44] J. Hisano, K. Ishiwata and N. Nagata, Gluon contribution to the dark matter direct detection, Phys. Rev. D 82 (2010) 115007 [arXiv:1007.2601] [INSPIRE].

[45] J. Hisano, K. Ishiwata and N. Nagata, QCD Effects on Direct Detection of Wino Dark Matter, JHEP 06 (2015) 097 [arXiv: 1504.00915] [INSPIRE].

[46] R.N. Mohapatra and R.E. Marshak, Local B-L Symmetry of Electroweak Interactions, Majorana Neutrinos and Neutron Oscillations, Phys. Rev. Lett. 44 (1980) 1316 [Erratum ibid. 44 (1980) 1643] [INSPIRE].

[47] W. Chao, H.-k. Guo and Y. Zhang, Majorana Dark matter with B $+L$ gauge symmetry, JHEP 04 (2017) 034 [arXiv:1604.01771] [INSPIRE]. 
[48] W. Chao, Phenomenology of the gauge symmetry for right-handed fermions, Eur. Phys. J. C 78 (2018) 103 [arXiv: 1707. 07858] [INSPIRE].

[49] T. Abe and R. Sato, Quantum corrections to the spin-independent cross section of the inert doublet dark matter, JHEP 03 (2015) 109 [arXiv:1501.04161] [INSPIRE].

[50] Spin Muon collaboration, A New measurement of the spin dependent structure function g1(x) of the deuteron, Phys. Lett. B 357 (1995) 248 [INSPIRE].

[51] H.H. Patel, Package-X: A Mathematica package for the analytic calculation of one-loop integrals, Comput. Phys. Commun. 197 (2015) 276 [arXiv:1503.01469] [inSPIRE].

[52] H.H. Patel, Package-X 2.0: A Mathematica package for the analytic calculation of one-loop integrals, Comput. Phys. Commun. 218 (2017) 66 [arXiv:1612.00009] [INSPIRE].

[53] V.A. Novikov, M.A. Shifman, A.I. Vainshtein and V.I. Zakharov, Calculations in External Fields in Quantum Chromodynamics. Technical Review, Fortsch. Phys. 32 (1984) 585 [INSPIRE].

[54] V. Shtabovenko, R. Mertig and F. Orellana, New Developments in FeynCalc 9.0, Comput. Phys. Commun. 207 (2016) 432 [arXiv:1601.01167] [INSPIRE].

[55] F. D'Eramo and M. Procura, Connecting Dark Matter UV Complete Models to Direct Detection Rates via Effective Field Theory, JHEP 04 (2015) 054 [arXiv:1411.3342] [INSPIRE].

[56] R.J. Hill and M.P. Solon, Standard Model anatomy of WIMP dark matter direct detection I: weak-scale matching, Phys. Rev. D 91 (2015) 043504 [arXiv:1401.3339] [INSPIRE].

[57] R.J. Hill and M.P. Solon, Standard Model anatomy of WIMP dark matter direct detection II: QCD analysis and hadronic matrix elements, Phys. Rev. D 91 (2015) 043505 [arXiv: 1409.8290] [INSPIRE].

[58] K.A. Mohan, D. Sengupta, T.M.P. Tait, B. Yan and C.P. Yuan, Direct Detection and LHC constraints on a t-Channel Simplified Model of Majorana Dark Matter at One Loop, JHEP 05 (2019) 115 [arXiv: 1903.05650] [INSPIRE].

[59] PandaX-II collaboration, Spin-Dependent Weakly-Interacting-Massive-Particle-Nucleon Cross section Limits from First Data of PandaX-II Experiment, Phys. Rev. Lett. 118 (2017) 071301 [Erratum ibid. 120 (2018) 049902] [arXiv: 1611.06553] [INSPIRE].

[60] XENON collaboration, Constraining the spin-dependent WIMP-nucleon cross sections with XENON1T, Phys. Rev. Lett. 122 (2019) 141301 [arXiv:1902.03234] [INSPIRE].

[61] G. Bélanger, F. Boudjema, A. Goudelis, A. Pukhov and B. Zaldivar, MicrOMEGAs5.0: Freeze-in, Comput. Phys. Commun. 231 (2018) 173 [arXiv:1801.03509] [inSPIRE].

[62] J. Pumplin, D.R. Stump, J. Huston, H.L. Lai, P.M. Nadolsky and W.K. Tung, New generation of parton distributions with uncertainties from global QCD analysis, JHEP 07 (2002) 012 [hep-ph/0201195] [INSPIRE].

[63] ATLAS collaboration, Constraints on mediator-based dark matter and scalar dark energy models using $\sqrt{s}=13 \mathrm{TeV}$ pp collision data collected by the ATLAS detector, JHEP 05 (2019) 142 [arXiv: 1903.01400] [INSPIRE].

[64] M. Chala, F. Kahlhoefer, M. McCullough, G. Nardini and K. Schmidt-Hoberg, Constraining Dark Sectors with Monojets and Dijets, JHEP 07 (2015) 089 [arXiv:1503.05916] [INSPIRE].

[65] F. Kahlhoefer, K. Schmidt-Hoberg, T. Schwetz and S. Vogl, Implications of unitarity and gauge invariance for simplified dark matter models, JHEP 02 (2016) 016 [arXiv: 1510.02110] [INSPIRE]. 